\title{
COUPLING ANT COLONY OPTIMIZATION AND DISCRETE-EVENT SIMULATION TO SOLVE A STOCHASTIC LOCATION-ROUTING PROBLEM
}

\author{
Nilson Herazo-Padilla ${ }^{1,2}$ \\ Jairo R. Montoya-Torres ${ }^{1}$ \\ Andrés Muñoz-Villamizar ${ }^{1}$ \\ ${ }^{1}$ Escuela Internacional de Ciencias Económicas y \\ Administrativas \\ Universidad de La Sabana \\ Chía (Cundinamarca), COLOMBIA
}

\author{
Santiago Nieto Isaza ${ }^{2}$ \\ Luis Ramirez Polo ${ }^{2}$ \\ ${ }^{2}$ Fundación Centro de Investigación en Modelación \\ Empresarial del Caribe (FCIMEC) \\ Barranquilla, COLOMBIA
}

\begin{abstract}
This paper considers the stochastic version of the location-routing problem (SLRP) in which transportation cost and vehicle travel speeds are both stochastic. A hybrid solution procedure based on Ant Colony Optimization (ACO) and Discrete-Event Simulation (DES) is proposed. After using a sequential heuristic algorithm to solve the location subproblem, ACO is employed to solve the corresponding vehicle routing problem. DES is finally used to evaluate such vehicle routes in terms of their impact on the expected total costs of location and transport to customers. The approach is tested using random-generated data sets. because there are no previous works in literature that considers the same stochastic location-routing problem, the procedure is compared against the deterministic version of the problem. Results show that the proposed approach is very efficient and effective.
\end{abstract}

\section{INTRODUCTION}

According to well-known definitions available in the literature (Simchi-Levy, Kaminsky, and SimchiLevy 2004; Melo, Nickel, and Saldanha-da-Gama 2009), Supply Chain Management (SCM) is "the process of planning, implementing and controlling the operations of the supply chain in an effective way. SCM spans all movements and storage of raw material, work-in-process inventory, and finished goods from the point-of-origin to the point-of-consumption". Part of the planning process in SCM aims to find the best possible configuration of the supply chain. In addition to the generic facility location problem, other areas (e.g., procurement, production, inventory, distribution, routing) has to be considered as well (Cordeau, Pasin, and Solomon 2006). These areas can be considered either as an integrated decisionmaking model or as independent, yet interconnected, models. The former approach drives to a very complex model, while the latter is easier to understand and to implement in real practice, yet requiring consistency between solutions of each decision level. 
In this paper, we consider an integrated supply chain design and operation problem concerned with facility location and vehicle routing decision-making. The problem is known in the literature as the Location-Routing Problem (LRP). The LRP is also viewed in the scientific literature as a research area within Location Theory, with the distinguishing property of paying special attention to underlying issues of vehicle routing (Nagy and Salhi 2007). The LRP is more complex than the traditional facility location problem (FLP) and more complex than the vehicle routing problem (VRP). The LRP hence belongs to the class of NP-hard problems, which means that it is not possible to find optimal solutions for large-sized instances in a reasonable computational time.

As explained in the next section, most of research works related to LRP studies the deterministic version. However, practitioners have to deal in fact with uncertainties real-life situations. This paper considers the stochastic location-routing problem (SLRP) in which transportation costs and vehicle travel speeds are both stochastic. To the best of our knowledge, this is the first time in literature that these stochastic characteristics are considered in SLRP. Because of its NP-completeness, a heuristic solution procedure is proposed. It is a hybrid structure based on sequential selection of depot locations, Ant Colony Optimization for vehicle routing and Discrete-Event Simulation to evaluate the system's stochastic behaviour.

The rest of this paper is organised as follows. Section 2 gives more insights about the problem under study and presents an overview of solution procedures existing in the literature. Section 3 is devoted to describe the proposed solution approach based on optimization and discrete-event simulation. Simulation experiments using random data is presented in Section 4. The paper ends in Section 5 by presenting some concluding remarks and opportunities for further research.

\section{THE (STOCHASTIC) LOCATION-ROUTING PROBLEM}

\subsection{Problem Statement}

Formally speaking, The LRP consists on both determining the locations of a set of depots from several candidates and finding the optimal set of vehicle routes (Srivastava 1993; Tuzun and Burke 1999; Tai, Low, and Bai 2002), as shown in Figure 1. LRP hence consists of two sub-problems: a location-allocation problem (LAP) and a vehicle routing problem (VRP) (Tai, Low, and Bai 2002). The former consists on choosing the best locations for depots from a given set of candidate sites in order to minimise the sum of the depot opening cost and the cost of assigning customers to the depots. The latter aims at determining the optimal delivery routes from a given depot to some geographically dispersed customers (Lui and Lee 2003).

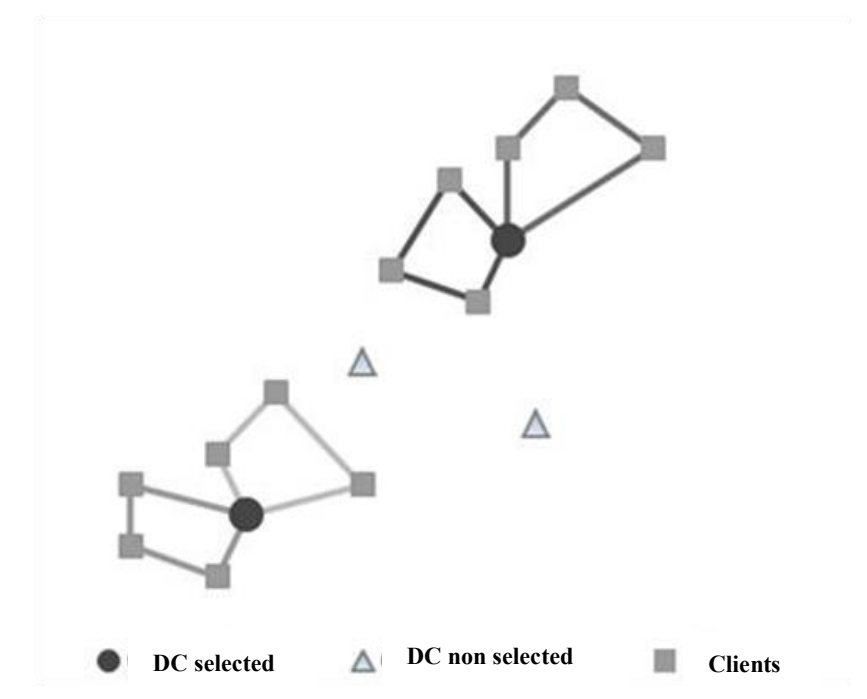

Figure 1: Graphical representation of a LRP. 
It is well known that facility location and vehicle routing are two interrelated problems. As a matter of fact, practitioners are very often concerned by making suboptimal decisions when depot location and vehicle routing decisions are made separately. Salhi and Nagy (2007) list three possible reasons that allow both academics and practitioners to often ignore this interrelation:

1. There are many practical situations when location problems do not have a routing issue. In these cases, the location-routing approach is clearly not an appropriate one.

2. Some researchers object to location-routing on the basis of a perceived inconsistency. They point out that location is a strategic, while routing is a tactical or even operational problem: routes can be re-calculated and re-drawn frequently (even daily), depot locations are normally for a much longer period. Thus, they claim that it is inappropriate to combine location and routing in the same planning framework due to their different planning horizons. This criticism led the authors to investigate this issue: it was found that the use of location-routing could decrease costs over a long planning horizon, within which routes are allowed to change (for a more detailed discussion on this issue, see Salhi and Nagy (1999)).

3. The LRP is conceptually more difficult than the classical location problem.

\subsection{Overview of Previous Solution Methods}

Some researchers consider that the first work published in the scientific literature about location-routing is that of Maranzana (1964), although strictly speaking it incorporates shortest-path, rather than vehiclerouting, problems into a location problem (Nagy and Salhi 2007). The location-routing problem (LRP) has received relatively little attention from researchers. State of the art surveys are presented by Balakrishnan, Ward, and Wong (1987), Laporte (1988, 1989), Berman, Jaillet, and Simchi-Levi (1995), Min, Jayaraman, and Srivastava (1998), and Nagy and Salhi (2007). Research has moved on considerably since these works.

Most of the research has focused on the deterministic version. Nagy and Salhi (2007) classifies the different solution heuristic methodologies for the (deterministic) LRP as sequential procedures, clustering-based, iterative and hierarchical. Sequential methods first solve the location problem and then the routing problem. This concept does not allow iterative interaction between both solutions. Srivastava and Benton (1990) claim that these procedures can give very good solutions and can be used as benchmarks for other heuristics. Clustering-based methods begin by partitioning the customer set into clusters: one cluster per potential depot or one per vehicle route. Then, they may proceed in two different ways: (a) by locating a depot in each cluster and then solving a VRP (or TSP) for each cluster; or (b) by solving a TSP for each cluster and then locating the depots. Iterative heuristics decompose the problem into its two constituent subproblems. Then, the methods iteratively solve the subproblems, feeding information from one phase to the other. Finally, hierarchical heuristics are algorithms where the main algorithm is devoted to solve the location problem and refers in each step to a subroutine that solves the routing problem.

Regarding the stochastic location-routing problem (SLRP), although taking uncertainty into account presents additional difficulties, there are a large number of papers of the stochastic LRP (Nagy and Salhi 2005). Most of these consider the special case of one depot and one vehicle (known as the travelling salesman location problem). We also note that most papers consider customer demand as the single input subject to stochastic variation. Our literature review revelled that about only $30 \%$ of works present an exact solution approach, while about $70 \%$ of published works propose an approximate algorithm. Among exact procedures for SLRP, we found multi-stage stochastic programming, linear programming models, and branch-and-bound algorithms. Among the set of currently available meta-heuristic procedures, the most employed have been Simulated Annealing, Genetics Algorithms, Tabu Search, Randomized Extended Clarke \& Wright Algorithm (RECWA) and Particle Swarm Optimization. 


\subsection{Contribution of this Paper}

It is to note, in the first instance, that among the solution procedures, Ant Colony Optimization has not been extensively used to solve stochastic location-routing problems. In addition, Discrete-Event Simulation can be regarded as a powerful tool to deal with some stochastic behaviour in SLRPs that is not easy to formalise into a complex meta-heuristic algorithm. Our approach intends to couple these two approaches to solve the SLRP with stochastic transportation costs and stochastic vehicle travel speed.

\section{SOLUTION APPROACH}

Because of the computational complexity of the stochastic location-routing problema (SLRP), this paper proposes an approximate solution based on solving two subproblems (see Figure 2). In the first instance, the problem of locating vehicle distribution centres (depots.) is solved. This is done through a sequential analysis of the impact of the location decision on both the vehicle routing (second phase of the procedure) and on the expected routing cost (third phase of the procedure). In the second phase, the routing problem is solved, that is the sequence to visit the clients is determined. The model employed here is the Capacitated Vehicle Routing Problem (CVRP). In the third phase, a discrete-event simulation model is built. Here, all stochastic elements are taken into account (transportation costs, vehicle speed, etc.). The final solution is obtained by comparing the performance of each location decision (found in the first phase) together with the sequence of visiting clients (found in the second phase).

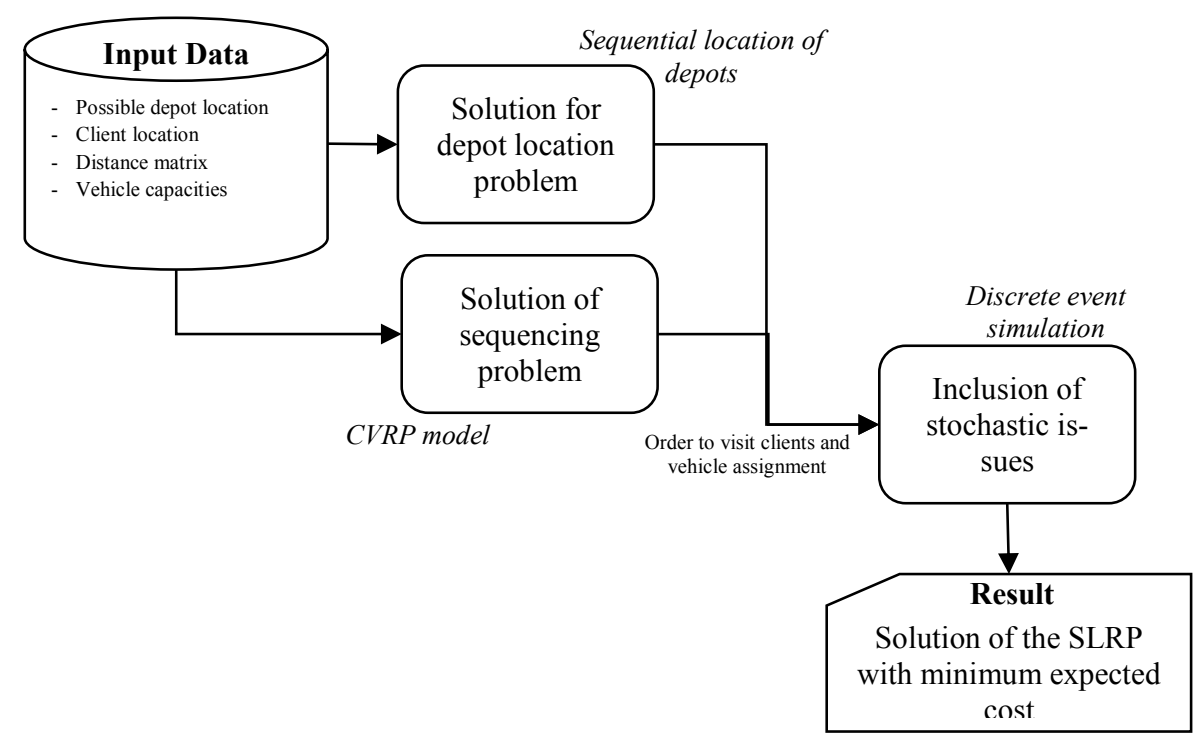

Figure 2: Approximate solution approach.

\subsection{Depot Location Problem}

The problem of determining the location of vehicle depot is solved using a random selection strategy. The selected depot is then considered as an input for the next steps of the procedures. For example, if there are five possible locations, one depot is first selected to solve the vehicle routing (second phase) and the computation of expected total transportation cost using simulation (third phase in the procedure). Them a second depot is randomly selected, and so on. Hence, as for this example, there will be five different evaluations of system performance using each of the possible depots at a time. 


\subsection{Sequencing Problem: Solving the Vehicle Routing Problem}

The problem of determining the order in which clients are to be visited by the vehicles is modelled as a capacitated Vehicle Routing Problem (CVRP). The CVRP can be represented using a directed graph $G=(A, V)$, where $V=\{0,1, \ldots, n\}$ represents the set of nodes (clients) and $A$ is the set of arcs connecting nodes. There is also a set $M=\{0,1, \ldots, m\}$ of vehicles located at the initial time at the depot. In a CVPR, the depot is denoted as node $j=0$, and clients are nodes $j=1,2, \ldots, n$, each one with a demand $d_{j}>0$. The set of arcs represents a possible route between nodes $i$ and $j$. Each arc has a weight representing the cost from going from node $i$ to node $j$, denoted as $C_{i j}>0$. If $C_{i j}=C_{j i}$ then the CVRP is known to be symmetric, otherwise, it is asymmetric. From the computational complexity standpoint, the CVRP is known to be a NP-hard problem, which means that is not possible to find optimal solutions for large-sized instances in a reasonable computational time. Because of this complexity, most of realistic problem instances have been solved using approximate algorithms, that is heuristics and meta-heuristics procedures.

For the purpose of this research an Ant Colony Optimization (ACO) algorithm is designed (Dorigo and Gambardella 1997; Bell and Griffis 2010). ACO algorithm emulates the behaviour of real ants when going from their nest to a source of food. ACO algorithms have been extensively designed to solve different types of vehicle routing problems. A pheromone trail is produce by each ant on its search of food, allowing the other ants to know which path is the shortest one to attain the objective. Pheromone evaporates over time, meaning that such paths are less interesting to ants in their search of food.

For the design of the ACO algorithm for the problem under study (see Figure 3), each ant represents a vehicle that builds its distribution route from the list of possible nodes (clients) to visit. Selection of clients is made until the vehicle capacity is not violated. Clients are assigned to vehicles giving priority to those routes with best balance between pheromone level and distance. The algorithm allows a random assignment of clients in order to avoid local optima. The selection criterion is determined using the wellknown formula presented by Dorigo and Gambardella (1997): $j=\arg \max \left(\left(\tau_{i j}\right)\left(1 / d_{i j}\right)^{\beta}\right)$, as soon as a random number $q$ is lower than a parameter $q_{0}$ allowing a random behaviour of the ant. $\tau_{i j}$ measures the level of pheromone on an arc and $d_{i j}$ is the distance between nodes $i$ and $j$. In the equation, the higher the value of parameter $\beta$, the lower the importance of distance and hence the higher the importance of the pheromone trail. In the cases in which $q$ is higher than $q_{0}$, then the next client is chosen by introduction a random number $s$.

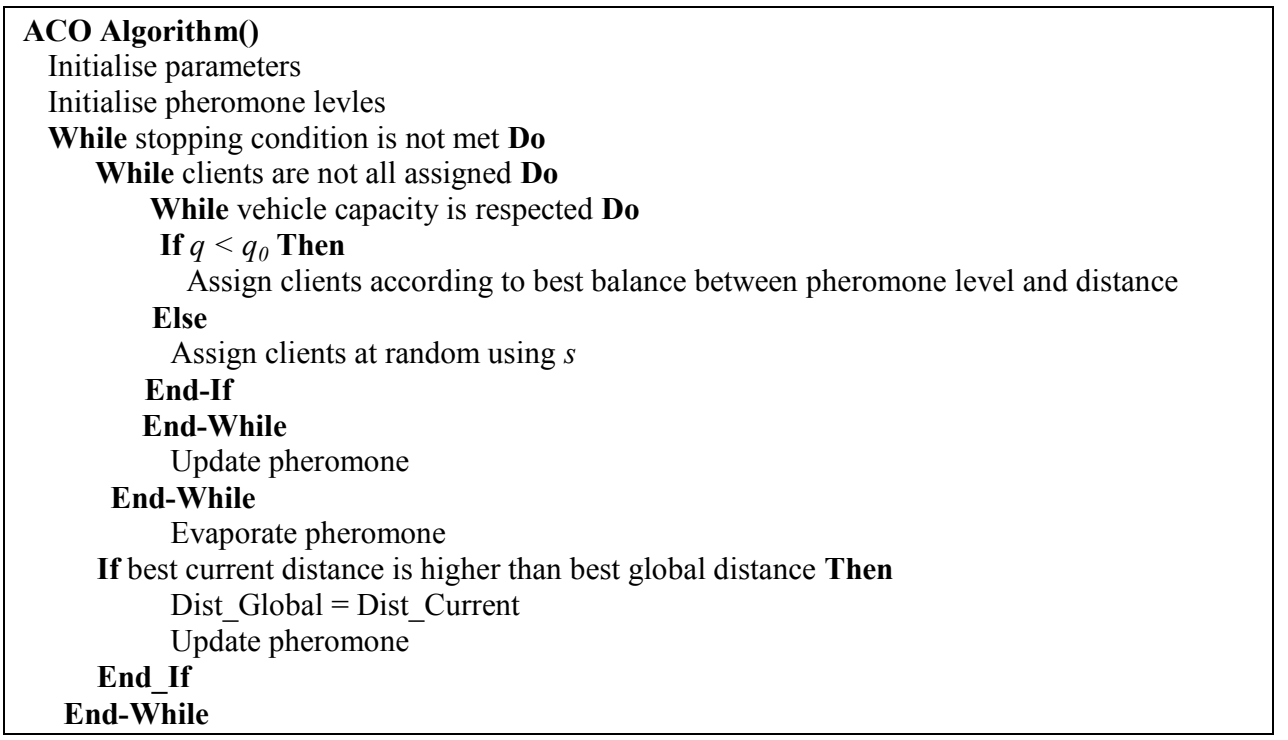

Figure 3: Proposed ACO algorithm pseudo-code. 
Local and global update of pheromone trail after selecting the current route is done by simulating the evaporation of pheromone using Equations (1) and (2):

and

$$
\tau_{I J}=(1-\alpha) \tau_{I J}+\alpha \tau_{0}
$$

$$
\tau_{I J}=(1-\alpha) \tau_{I J}+\alpha L^{-1}
$$

where $\tau_{0}$ is an initial value of pheromone trail and $\alpha$ is the level of evaporation. The first update is applied to all arcs while the second is only applied to those arc belonging to the best route found. $L$ is the best distance found.

In order to set up the parameters of ACO algorithm, we used the same values defined by Bell and Griffis (2010). Such values are $\alpha=0.1, \beta=2.3$ y $q_{0}=0.9$. Small instances were run in order to validate the algorithm behaviour. We found optimal values for the sets of "Solomon Instances" with 5 and 10 clients. We also carried out preliminary runs with a set of ten instances presented by Bell and Griffis (2010) in which 20 clients are to be served and vehicles have a total capacity of 100 demand units. Table 1 presents a summary of best solutions available up to date, as well as the ones given by ACO and those obtained using the well-known Cost-Savings Algorithm. It is to note that the higher computational time of the proposed ACO algorithm is about 8.81 seconds, while the average execution time over all instances is 7 seconds. Finalmente se tienen resultados aceptables con una desviación que oscila alrededor del $7 \%$ considerando que el algoritmo presentado no incluye una fase de mejoramiento de las mejores rutas encontradas.

\begin{tabular}{|c|c|c|c|c|c|c|c|c|c|c|}
\hline & \multicolumn{10}{|c|}{ Instance } \\
\hline & 1 & 2 & 3 & 4 & 5 & 6 & 7 & 8 & 9 & 10 \\
\hline $\begin{array}{c}\text { Best } \\
\text { known }\end{array}$ & 444.14 & 457.38 & 454.88 & 513.08 & 501.37 & 566.77 & 529.64 & 536.57 & 541.85 & 475.45 \\
\hline CSA & 484 & 503 & 459 & 522 & 516 & 593 & 530 & 591 & 557 & 477 \\
\hline ACO best & 443.26 & 456.80 & 454.88 & 477.80 & 501.37 & 566.77 & 538.22 & 537.93 & 548.32 & 475.45 \\
\hline$\% \mathrm{dev}$ & $0 \%$ & $0 \%$ & $0 \%$ & $-0.07 \%$ & $0 \%$ & $0 \%$ & $0.02 \%$ & $0 \%$ & $0.01 \%$ & $0 \%$ \\
\hline ACO avg & 459.38 & 456.80 & 454.88 & 509.87 & 501.64 & 573.48 & 538.22 & 537.93 & 550.12 & 475.45 \\
\hline
\end{tabular}

Table 1: Best solutions for instances used to validate the proposed ACO algorithm.

\subsection{Computing the Expected Routing Cost}

The total transportation cost is computed using a discrete-event simulation model in order to incorporate stochasticity regarding vehicle speed and transportation costs. The model was built using the commercial software ARENA®. Figure 4 presents the logical structure of the simulation model. 
Herazo-Padilla, Nieto Isaza, Montoya-Torres, Ramírez Polo, and Muñoz-Villamizar

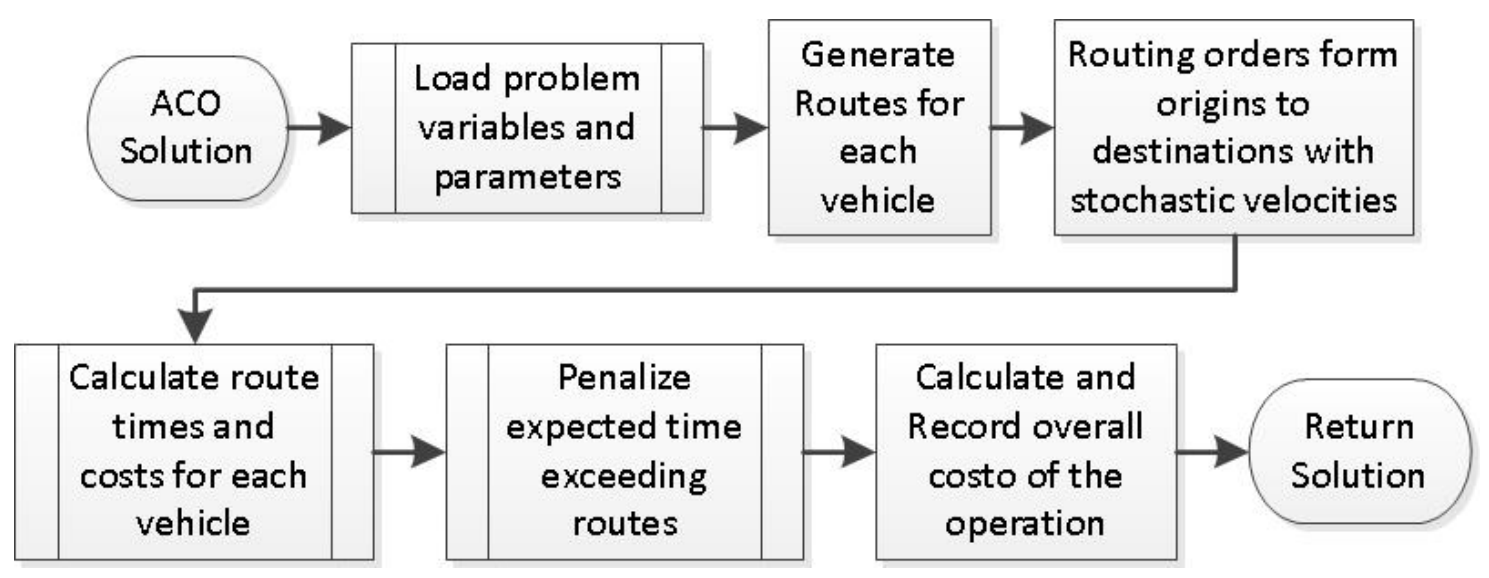

Figure 4: Examples of modules in the simulation model.

\section{EXPERIMENTS}

Computational experiments were carried out using a total of 40 random-generated instances. These instances have $5,10,20$ or 50 clients and a total of 5 possible locations for depots. For each combination and number of possible depots, a total of 10 different instances were generated. Vehicle capacity was fixed to be 200 demand units. Demands were modelled using a uniform distribution between 1 and 50 units. Vehicle speed is modelled using a normal distribution with mean $60 \mathrm{~km}$ per hour and standard deviation $7 \mathrm{~km}$ per hour. These instances were run on a PC Intel(R) Core(TM) i7 - 2670QM CPU @ 2.20GHz, with $8.00 \mathrm{~GB}$ of RAM and Windows 7 Home Premium operating system.

For the design of experiments, both a deterministic and a stochastic version of the problem were run. For the latter, a total of 10 replications were run. A summary of results over the complete set of instances in presented in Tables 2 and 3. Table 2 presents the average values of the percentage deviation of the stochastic cost of route against the cost if the problem were deterministic. This deviation is computed using Equation (3):

$$
\% d e v=\frac{\text { Total expected } \operatorname{cost}(\text { stochastic })-\text { Total } \operatorname{cost}(\text { deterministic })}{\text { Total } \operatorname{cost}(\text { deterministic })} \times 100 \%
$$

Cells in gray corresponds to the lowest expected total cost obtained using simulation among the five different depot locations (that is, solution of the stochastic LRP). The actual values of this costs are presented in Table 3.

In Table 2 we present the total expected cost percentage of deviation for each specific solution, generated to solve the stochastic LRP in each instance, as we highlight in every case the selected central depot (the one with the smaller expected cost). For example, in instance I1-5 which presents a problem with 5 nodes, the central depot that presented the best performance was the number 4 , in turn, the one with best performance in instance I1-10 was depot number 5 and so forth. We found that in about $30 \%$ of the cases the central depot with the best performance was also the one with the smaller percentage of deviation of the stochastic problem regarding the deterministic version. Among the others central depots that presented the bests performances we found similar percentages of deviation as well as values very closed to the smaller one. Some exceptional cases were also found such as the solution to instance I3-20 where although the greater percentage of deviation of central depot 4 it presented the smaller expected cost. The experiments reveal that considering the stochastic behaviour of location and routing problems generates mean deviations from $0,5 \%$ to $7 \%$ in the overall cost of the operation over not considering stochastic parameters existing among this kind of problems, i.e., when deterministic solutions are considered to solve 
the problem. Such deviations are quite significant taking into account that transport costs represent a notable expense for companies in their distribution operations. Thus, not considering those aspects could result in a poor estimation of costs which together with a poor estimation of delivery times could generate low service levels and quality deficiencies.

Table 3 presents the summary of expected costs for each specific solution generated to solve each instance. A total of 10 replications for each solution generated to every specific depot were run to ensure a level of confidence of $95 \%$ with a mean error that in most of the cases was $1 \%$ in average. It was found that the larger the instance solved the greater the difference between the costs of the solutions for each depot, namely, larger number of nodes correspond to major differences in costs among the five central depots evaluated. Differences between the solution from the central depot with the smaller overall expected cost and the one with higher overall expected cost of 10 up to $45 \%$ were observed corresponding greater average differences to instances with larger number of nodes. Results reveal that, as is well known, a miscalculation in a strategic decision making, such as a central depot location decision imply a substantial raise in the overall cost of the operation. Hence, regarding stochastic aspects of such problems would have a greater impact in the expected performance of the different potential locations. Therefore we highlight that modelling location decisions regarding the performance of routing operations, together with stochastic aspects of such kind of problems, could produce better estimations of costs and resources associated which results in improvements in quality and service levels as well as significant savings in distribution operations.

Table 2: Comparison between deterministic and stochastic version of the LRP.

\begin{tabular}{|c|c|c|c|c|c|c|c|c|c|c|c|c|}
\hline \multirow{2}{*}{$\begin{array}{l}\text { Nber. } \\
\text { Nodes }\end{array}$} & \multirow{2}{*}{ Depot } & \multicolumn{10}{|c|}{ Instance } & \multirow{2}{*}{ Avg. } \\
\hline & & I1 & $\mathrm{I} 2$ & $\mathrm{I} 3$ & I4 & I5 & I6 & 17 & I8 & I9 & $\mathrm{I} 10$ & \\
\hline \multirow{5}{*}{5} & D1 & $2,1 \%$ & $1,9 \%$ & $2,0 \%$ & $1,9 \%$ & $1,5 \%$ & $1,6 \%$ & $1,9 \%$ & $1,6 \%$ & $1,4 \%$ & $2,4 \%$ & $1,8 \%$ \\
\hline & $\mathrm{D} 2$ & $2,1 \%$ & $2,0 \%$ & $0,5 \%$ & $2,0 \%$ & $0,8 \%$ & $1,6 \%$ & $1,4 \%$ & $1,4 \%$ & $3,4 \%$ & $1,9 \%$ & $1,7 \%$ \\
\hline & D3 & $1,9 \%$ & $2,0 \%$ & $1,9 \%$ & $1,8 \%$ & $1,2 \%$ & $1,6 \%$ & $1,6 \%$ & $1,4 \%$ & $1,3 \%$ & $2,1 \%$ & $1,7 \%$ \\
\hline & D4 & $2,1 \%$ & $1,8 \%$ & $1,8 \%$ & $2,0 \%$ & $1,5 \%$ & $1,6 \%$ & $1,1 \%$ & $1,5 \%$ & $1,3 \%$ & $1,83 \%$ & $1,6 \%$ \\
\hline & D5 & $2,1 \%$ & $2,1 \%$ & $1,9 \%$ & $2,2 \%$ & $1,2 \%$ & $5,5 \%$ & $1,5 \%$ & $1,4 \%$ & $2,3 \%$ & $1,9 \%$ & $2,2 \%$ \\
\hline \multirow{5}{*}{10} & D1 & $3,0 \%$ & $1,1 \%$ & $0,8 \%$ & $1,1 \%$ & $0,9 \%$ & $0,8 \%$ & $0,9 \%$ & $1,1 \%$ & $0,9 \%$ & $1,1 \%$ & $1,2 \%$ \\
\hline & $\mathrm{D} 2$ & $0,6 \%$ & $3,8 \%$ & $0,9 \%$ & $0,8 \%$ & $1,0 \%$ & $0,8 \%$ & $1,1 \%$ & $3,8 \%$ & $9,5 \%$ & $1,3 \%$ & $2,4 \%$ \\
\hline & D3 & $1,0 \%$ & $1,3 \%$ & $0,9 \%$ & $1,2 \%$ & $1,1 \%$ & $0,7 \%$ & $0,7 \%$ & $0,9 \%$ & $0,7 \%$ & $1,3 \%$ & $1,0 \%$ \\
\hline & D4 & $0,9 \%$ & $1,4 \%$ & $3,0 \%$ & $1,4 \%$ & $0,9 \%$ & $0,8 \%$ & $1,0 \%$ & $1,0 \%$ & $0,7 \%$ & $1,3 \%$ & $1,3 \%$ \\
\hline & D5 & $1,0 \%$ & $2,3 \%$ & $0,9 \%$ & $1,2 \%$ & $0,9 \%$ & $1,0 \%$ & $1,2 \%$ & $1,0 \%$ & $0,9 \%$ & $0,9 \%$ & $1,1 \%$ \\
\hline \multirow{5}{*}{20} & D1 & $0,7 \%$ & $0,6 \%$ & $0,5 \%$ & $1,9 \%$ & $1,9 \%$ & $0,5 \%$ & $0,8 \%$ & $0,8 \%$ & $0,6 \%$ & $0,8 \%$ & $0,9 \%$ \\
\hline & $\mathrm{D} 2$ & $0,5 \%$ & $0,7 \%$ & $0,5 \%$ & $0,7 \%$ & $0,5 \%$ & $0,1 \%$ & $0,7 \%$ & $0,7 \%$ & $0,7 \%$ & $0,6 \%$ & $0,6 \%$ \\
\hline & D3 & $1,0 \%$ & $0,8 \%$ & $5,2 \%$ & $0,7 \%$ & $0,6 \%$ & $0,6 \%$ & $0,8 \%$ & $3,8 \%$ & $0,5 \%$ & $1,2 \%$ & $1,5 \%$ \\
\hline & $\mathrm{D} 4$ & $2,5 \%$ & $0,7 \%$ & $6,4 \%$ & $2,4 \%$ & $0,7 \%$ & $0,7 \%$ & $1,9 \%$ & $0,7 \%$ & $0,6 \%$ & $0,8 \%$ & $1,7 \%$ \\
\hline & D5 & $5,3 \%$ & $0,7 \%$ & $0,7 \%$ & $0,1 \%$ & $0,7 \%$ & $0,6 \%$ & $1,0 \%$ & $0,6 \%$ & $0,5 \%$ & $0,8 \%$ & $1,1 \%$ \\
\hline \multirow{5}{*}{50} & D1 & $2,4 \%$ & $0,3 \%$ & $0,3 \%$ & $0,2 \%$ & $0,3 \%$ & $2,1 \%$ & $0,3 \%$ & $0,3 \%$ & $0,7 \%$ & $1,2 \%$ & $0,8 \%$ \\
\hline & D2 & $0,4 \%$ & $0,3 \%$ & $0,7 \%$ & $0,3 \%$ & $0,3 \%$ & $5,8 \%$ & $0,4 \%$ & $0,2 \%$ & $0,3 \%$ & $0,3 \%$ & $0,9 \%$ \\
\hline & D3 & $0,3 \%$ & $0,5 \%$ & $1,2 \%$ & $0,9 \%$ & $0,3 \%$ & $2,3 \%$ & $0,4 \%$ & $0,3 \%$ & $0,3 \%$ & $0,3 \%$ & $0,7 \%$ \\
\hline & D4 & $0,3 \%$ & $0,3 \%$ & $0,3 \%$ & $0,3 \%$ & $0,2 \%$ & $0,3 \%$ & $0,3 \%$ & $0,3 \%$ & $0,3 \%$ & $0,3 \%$ & $0,3 \%$ \\
\hline & D5 & $0,6 \%$ & $0,2 \%$ & $0,5 \%$ & $7,5 \%$ & $0,3 \%$ & $4,3 \%$ & $0,4 \%$ & $0,4 \%$ & $0,3 \%$ & $0,3 \%$ & $1,5 \%$ \\
\hline
\end{tabular}


Herazo-Padilla, Nieto Isaza, Montoya-Torres, Ramírez Polo, and Muñoz-Villamizar

Table 3: Expected cost for the SLRP among the set of possible depots.

\begin{tabular}{|c|c|c|c|c|c|c|c|c|}
\hline & \multicolumn{2}{|c|}{5 nodes } & \multicolumn{2}{|c|}{10 nodes } & \multicolumn{2}{|c|}{20 nodes } & \multicolumn{2}{|c|}{50 nodes } \\
\hline & $\begin{array}{c}\text { Expected } \\
\text { cost }\end{array}$ & $\begin{array}{c}\text { Best de- } \\
\text { pot }\end{array}$ & $\begin{array}{c}\text { Expected } \\
\text { cost }\end{array}$ & $\begin{array}{c}\text { Best de- } \\
\text { pot }\end{array}$ & $\begin{array}{l}\text { Expected } \\
\text { cost }\end{array}$ & $\begin{array}{c}\text { Best de- } \\
\text { pot }\end{array}$ & $\begin{array}{c}\text { Expected } \\
\text { cost }\end{array}$ & $\begin{array}{c}\text { Best de- } \\
\text { pot }\end{array}$ \\
\hline I1 & $368.913,21$ & D4 & $626.374,89$ & D5 & $988.541,77$ & D5 & $2.765 .943,47$ & $\mathrm{D} 4$ \\
\hline I2 & $293.368,15$ & D1 & $481.293,99$ & $\mathrm{D} 1$ & $1.161 .436,81$ & D1 & $2.503 .846,03$ & D1 \\
\hline $\mathrm{I} 3$ & $367.000,93$ & D5 & $749.474,00$ & $\mathrm{D} 2$ & $1.422 .496,97$ & D4 & $1.966 .563,53$ & $\mathrm{D} 2$ \\
\hline I4 & $271.695,36$ & D2 & $666.615,05$ & D4 & $1.039 .961,22$ & D3 & $2.427 .844,81$ & D1 \\
\hline I5 & $410.081,97$ & $\mathrm{D} 2$ & $681.683,90$ & D1 & $1.120 .941,81$ & D5 & $2.635 .377,24$ & D4 \\
\hline I6 & $489.400,93$ & D2 & $747.896,29$ & D5 & $1.313 .411,37$ & D1 & $2.570 .861,54$ & D3 \\
\hline I7 & $346.556,29$ & D3 & $657.953,98$ & D5 & $978.877,97$ & $\mathrm{D} 2$ & $2.403 .209,83$ & D1 \\
\hline I8 & $465.824,60$ & D4 & $661.158,01$ & D1 & $923.442,16$ & D1 & $2.503 .152,77$ & D3 \\
\hline I9 & $474.752,67$ & D1 & $628.183,94$ & D1 & $870.793,52$ & D4 & $2.639 .117,63$ & $\mathrm{D} 4$ \\
\hline $\mathrm{I} 10$ & $273.390,95$ & D1 & $593.461,91$ & $\mathrm{D} 2$ & $928.089,73$ & D1 & $2.975 .972,93$ & D5 \\
\hline
\end{tabular}

\section{CONCLUSIONS AND PERSPECTIVES}

This paper presented a sequential approximation algorithm to solve the stochastic location-routing problem (SLRP). The proposed procedure employs Ant Colony Optimization (ACO) algorithm to solve the sequencing problem for visit clients in the distribution network. Then, the routing solution is used as input in a simulation model that is able to use stochastic data related to transportation costs and vehicle speed. Hence, the expected total transportation cost is computed. The final location decision is made by comparing all expected costs. Experiments were carried out using random-generated data. Results showed the benefits that can be achieved using this approach.

For further works, we plan to continue this research in two main directions. In the first instance, we will compare the performance of this hybrid ACO-Simulation approach with an exact method, especially regarding the routing decision level. In a second step, our research will solve a real-life distribution problem for a Colombian company that transport hazardous material. This last further research line is extremely relevant as the work presented here focused mainly on the approach to solve a relevant stochastic problem in transportation, rather than exploiting the algorithms in a commercial tool. The industrial application will allow us to validate this approach in a realistic context and hence aid in the future design of a decision-support tool based on simulation-optimization.

\section{ACKNOWLEDGMENTS}

This research was supported by the Colombian Department of Science, Technology and Innovation (Colciencias) under Grant No. 333-502-27900. Part of this paper was written while Jairo R. Montoya-Torres was Visiting Scholar at Leeds University Business School, University of Leeds, UK, supported by a Marie Curie International Incoming Fellowship within the 7th European Community Framework Programme (project “DISRUPT”, grant No. ESR-299255).

\section{REFERENCES}

Balakrishnan, A., J.E. Ward, and R.T. Wong. 1987. "Integrated facility location and vehicle routing models: recent work and future prospects." American Journal of Mathematical and Management Sciences 7:35-61. 
Bell, J., and S. Griffis. 2010. "Swarm Intelligence: Application of the Ant Colony Optimization Algorithm to Logistics-Oriented Vehicle Rioting Problem." Journal of Business Logistics 31(2):157-175.

Berman, O., P. Jaillet, and D. Simchi-Levi. 1995. "Location-routing problems with uncertainty." In Facility Location: A Survey of Applications and Methods, Edited by Z. Drezner, 427-452. New York, New York: Springer

Cordeau, J.-F., F. Pasin, and M.M. Solomon. 2006. "An integrated model for logistics network design." Annals of Operations Research 144(1):59-82.

Laporte, G. 1988. "Location-routing problems." In Vehicle Routing: Methods and Studies, Edited by B.L. Golden, and A.A. Assad, 163-198. Amsterdam: North-Holland.

Laporte, G. 1989. “A survey of algorithms for location-routing problems." Investigación Operativa 1:93123.

Liu, S.C., and S. B. Lee. 2003. "A two-phase heuristic method for the multi-depot location routing problem taking inventory control decisions into consideration." International Journal of Advanced Manufacturing Technology 22:941-950.

Maranzana, F.E. 1964. "On the location of supply points to minimise transport costs." Operational Research Quarterly 15:261-270.

Melo, M.T., S. Nickel, and F. Saldanha-da-Gama. 2009. "Facility location and supply chain management - A review." European Journal of Operational Research 196(2):401-412.

Min, H., V. Jayaraman, and R. Srivastava. 1998. "Combined location-routing problems: a synthesis and future research directions." European Journal of Operational Research 108:1-15.

Nagy, G., and S. Salhi. 2007. "Location-routing: Issues, models and methods." European Journal of Operational Research 177(2): 649-672

Salhi, S., and G. Nagy. 1999. "Consistency and robustness in location-routing." Studies in Locational Analysis 13:3-19.

Simchi-Levy, D., P. Kaminsky, and E. Simchi-Levy. 2004. Designing and managing the supply chain: Concepts, strategies, and cases. New York, New York: McGraw Hill.

Srivastava, R. 1993. "Alternate solution procedures for the location-routing problem." Omega 21:497506.

Tai, H.W., C. Low, and J.W. Bai. 2002. "Heuristic solutions to multidepot location-routing problems." Computers \& Operations Research 29:1393-1415.

Tuzun, D., and L.I. Burke. 1999. "A two-phase tabu search approach to the location routing problem." European Journal of Operational Research 166:87-99.

\section{AUTHOR BIOGRAPHIES}

NILSON HERAZO PADILLA is a Research Assistant within the School of Economics and Management Sciences at Universidad de La Sabana, Chia, Colombia and Junior Research at Fundación Centro de Investigación en Modelación Empresarial del Caribe (FCIMEC), Barranquilla, Colombia. He is pursuing the Master in Operations Management program at Universidad de La Sabana, Chía, Colombia, and holds a BSc in Industrial Engineering from Universidad de la Costa in Barranquilla, Colombia. His email address is ing.nilsonherazo@gmail.com.

SANTIAGO NIETO ISAZA is Associate Researcher at Fundación Centro de Investigación en Modelación Empresarial del Caribe (FCIMEC), Barranquilla, Colombia, and full time lecturer at Universidad de la Costa, Barranquilla, Colombia. His research interests include optimization of manufacturing systems, vehicle routing with multiple objectives and simulation. His email address is snieto@fcimec.org.

JAIRO R. MONTOYA-TORRES is Full Professor in the School of Economics and Management Sciences at Universidad de La Sabana, Chia, Colombia. He is currently Visiting Scholar (2013-2015) at the 
Leeds University Business School at University of Leeds, UK, under a Marie Curie International Incoming Fellowship. His research interests lie broadly in simulation and optimization of logistics and production systems, scheduling, and supply chain management under collaborative and sustainable environments. He is a member of several academic societies, including ACM and EUROSIS, and has served as Guest Editor or within the Editorial Board of various academic journals. He can be contacted by email at jairo.montoya@unisabana.edu.co. His web page is http://jrmontoya.wordpress.com.

LUIS RAMÍREZ POLO is the CEO of Fundación Centro de Investigación en Modelación Empresarial del Caribe (FCIMEC), in Barranquilla, Colombia, and full-time lecturer at Universidad Autónoma del Caribe, Barranquilla, Colombia. His research interests includes simulation of logistics systems and supply chains. His email address is 1ramirez@fcimec.org.

ANDRÉS MUÑOZ VILLAMIZAR is a Research Assistant within the School of Economics and Management Sciences at Universidad de La Sabana, Chia, Colombia. He is pursuing the Master in Operations Management program at the same university, and holds the Industrial Engineering degree from Escuela Colombiana de Ingeniería Julio Garavito, Bogotá, Colombia. His research has been focused on solving location-routing problems found in the configuration of urban logistic platforms. He can be contacted by email to the address andres_10_89@hotmail.com. 